\title{
Love and appeal in standard couples
}

Sergio Rinaldi (rinaldi@elet.polimi.it)

Fabio Della Rossa (dellarossa@elet.polimi.it)

Fabio Dercole (fabio.dercole@polimi.it)

\section{Approved by}

Ulf Dieckmann

Program Leader, EEP

July 2011

Interim Reports on work of the International Institute for Applied Systems Analysis receive only limited review. Views or opinions expressed herein do not necessarily represent those of the Institute, its National Member Organizations, or other organizations supporting the work. 


\section{Contents}

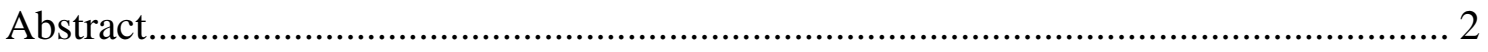



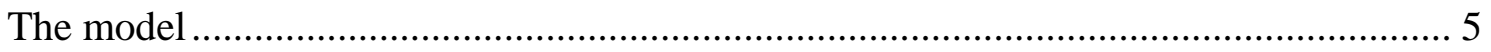

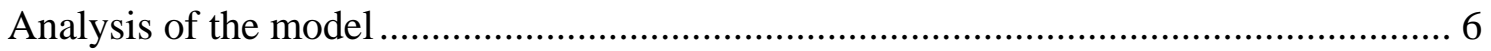

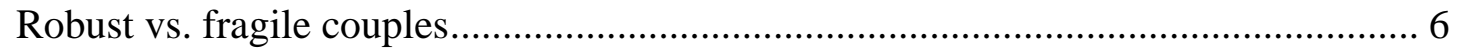

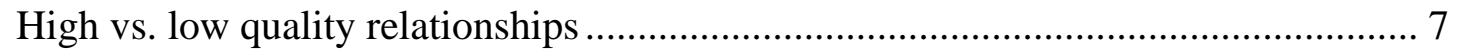

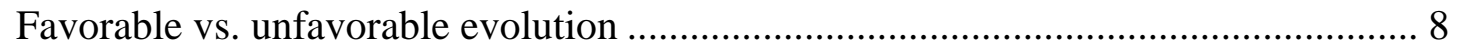



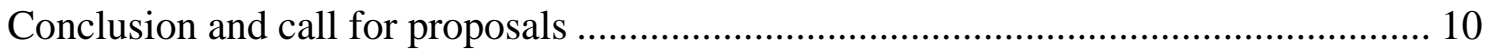

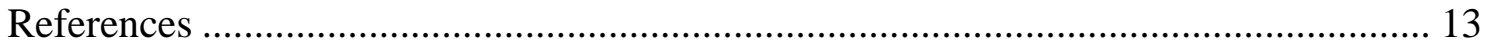



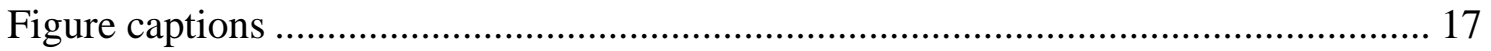

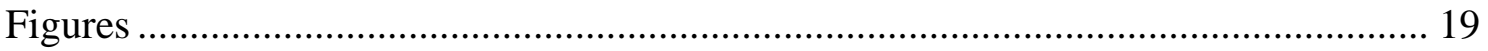




\title{
Love and appeal in standard couples
}

\author{
Sergio Rinaldi ${ }^{1-3}$, Fabio Della Rossa ${ }^{1}$, Fabio Dercole ${ }^{1}$
}

${ }^{1}$ DEI, Politecnico di Milano

Via Ponzio 34/5, 20133 Milano, Italy

${ }^{2}$ Evolution and Ecology Program, International Institute for Applied Systems Analysis 2361 Laxenburg, Austria

${ }^{3}$ To whom correspondence should be addressed Ph: +39 022399 3563; Fax: +39 0223993412

sergio.rinaldi@polimi.it 


\section{Abstract}

Through the analysis of a mathematical model we construct in this paper the full catalog of possible love stories among standard (i.e., secure and non-synergic) individuals. For this, we first distinguish between robust and fragile couples, i.e., couples which have one or two stable equilibria. We also distinguish between high and low quality relationships by looking at the sign of the feelings among the partners. Finally, we split fragile couples into two distinct classes, namely those that starting from the state of indifference evolve toward their most desirable equilibrium, as opposed to those that have an unfavorable evolution. The boundaries separating the various classes in any two dimensional parameter space are either bifurcation curves or special curves characterized by the annihilation of a suitably defined indicator associated to the equilibria or to their basins of attraction. The analysis is performed in part analytically and in part numerically (through continuation methods), and is mainly focused on the role of the appeals of the individuals. The results are in agreement with common wisdom and observations and show, in particular, that love stories can evolve positively even if one of the two individuals has negative feelings in the initial phase of the relationship.

Key words: Love dynamics, mathematical models, dynamical systems, bifurcation analysis, bistability, continuation method. 


\section{Introduction}

This paper deals with love dynamics, a subject falling in the field of social psychology, where interpersonal relationships are the topic of major concern. Romantic relationships are somehow the most simple case since they involve only two individuals.

Love-stories are dynamic processes that start from zero (two persons are completely indifferent one to each other when they first meet), develop (more or less quickly) and end up into a sort of regime. Real-life observations tell us that frequently transients develop quite regularly and asymptotic regimes are stationary and associated to positive romantic relationships. But observations also point out the existence of multiple attractors. For example, it is known that steady and high quality romantic relationships can turn into states

of permanent antagonism after a disturbance, for example after a temporary infatuation of one of the two partners for another person. This spontaneously suggests the use of differential equations for modeling the dynamics of the feelings between two individuals.

Although dynamic phenomena in physics, chemistry, economics, and all other sciences have been extensively studied by means of differential equations, surprisingly the first contribution in social psychology are two unpublished notes by Etienne Guyon and Steven Strogatz, written in 1975 and 1978, respectively, in which a very simple linear model for a generic couple is analyzed. The first published contribution is a one page paper in which Strogatz [1988] describes how the classical topic of harmonic oscillations can be taught to capture the attention of students. He suggests to make reference to "a topic that is already on the minds of many college students: the time evolution of a love affair between two people." The model proposed by Strogatz (discussed also in [Radzicki, 1993], [Strogatz, 1994], and [Sprott, 2004]) is definitely unrealistic because it does not take into account the appeals of the two individuals. Thus, Strogatz's model doesn't explain, for example, why two persons who are initially completely indifferent one to each other can develop a love affair.

By contrast, we focus in this paper on the effects of the appeals on the development of a love story. This is a quite important issue, because the appeals, which have a dominant 
role in the initial phase of any love story, can be strategically important also in the long run. Some results obtained through simple mathematical models, are already available along this line [Gragnani et al., 1997], [Rinaldi, 1998a,b], [Rinaldi \& Gragnani, 1998], [Wauer et al., 2007] but refer only to the case of positive appeals. Here we enlarge the scope of the analysis and deal also with the consequences of negative appeals. In particular, we like to answer questions like "Is it possible that love stories involving non appealing individuals develop positively after an initial phase of antagonism?" or, more crudely "Can individuals be happy even if they are not appealing?" Of course, it must be understood that appeal is not mere physical attractiveness, but, more properly and in accordance with evolutionary theory, a suitable combination of different attributes among which age, education, potential earning, and social position. Moreover, the relative weights that each individual gives to the above attributes strongly depend upon her/his character, so that it can easily be that a person is perceived as appealing by the partner and as not appealing by an alternative potential partner. In other words, the appeal is not an absolute characteristic value of an individual, but rather a subjective value given by the partner. Thus, given as granted that high appeals promote the development of high quality feelings in a couple, it is not so important to be ranked as appealing by the majority of the people in a given community, but rather to be appreciated by the partner. This obvious remark explains why individuals perceived as non appealing in their community can develop very positive love stories.

The analysis is limited to so-called standard couples composed of secure and non-synergic individuals. Loosely speaking, secure individuals are those who "love to be loved" and "hate to be hated" and therefore respond positively to any increase of the love of the partner [Bartholomew \& Horowitz, 1991], [Griffin \& Bartholomew, 1994]. In contrast, non-secure individuals typically reduce their reaction when pressure and involvement become too high. On the other hand, non-synergic individuals are those who respond to the love and appeal of the partner independently upon their state of involvement. A typical example of a synergic individual is a mother with a biased view of the beauty of her child. Synergism has 
been observed empirically in a study of perception of physical attractiveness [Simpson et al., 1990] by comparing individuals involved with individuals not involved in dating relationships. Synergism and insecurity are here excluded because they can give rise to love stories characterized by ups and downs [Gragnani et al., 1997].

The target of this study is to partition through bifurcation analysis [Kuznetsov, 2004] and continuation techniques[Allgower \& Georg, 1990], the class of standard couples into various subclasses characterized by the number of alternative stable states, their quality (identified by the signs of the feelings of the two individuals), and by the tendency to evolve toward the most desirable state from the state of indifference, where most couples are at the beginning of the love story.

\section{The Model}

The formal description of love dynamics in a couple requires at least two state variables, $x_{1}$ and $x_{2}$, representing the love of individuals 1 and 2 for the partner. In this paper we describe standard couples with the following model:

$$
\begin{aligned}
& \dot{x}_{1}=-\alpha_{1} x_{1}+R_{1}\left(x_{2}\right)+S_{1}\left(A_{2}\right), \\
& \dot{x}_{2}=-\alpha_{2} x_{2}+R_{2}\left(x_{1}\right)+S_{2}\left(A_{1}\right),
\end{aligned}
$$

where $\alpha_{i}$ and $A_{i}$ are the forgetting coefficient and the appeal of individual $i, R_{i}\left(x_{j}\right)$ and $S_{i}\left(A_{j}\right)$ are the reactions of individual $i$ to the love $x_{j}$ and the appeal $A_{j}$ of the partner. The coefficients $\alpha_{i}$ are assumed to be constant and positive, while the appeals are constant, but can be negative. The reaction functions $R(\cdot)$ and $S(\cdot)$ are smooth, increasing, and bounded, and change sign with their arguments (i.e., $\left.R_{i}(0)=S_{i}(0)=0\right)$. Figure 1 reports typical examples of reaction functions $R_{i}\left(x_{j}\right)$ of two secure and non-synergic individuals. For a discussion on the significance and limitations of the model the reader can refer to [Rinaldi \& Gragnani, 1998]. Since we will not discuss the influence of the reaction functions $S_{i}$ we 
will simply identify them with their arguments and discuss the following model:

$$
\begin{aligned}
& \dot{x}_{1}=-\alpha_{1} x_{1}+R_{1}\left(x_{2}\right)+A_{2}, \\
& \dot{x}_{2}=-\alpha_{2} x_{2}+R_{2}\left(x_{1}\right)+A_{1} .
\end{aligned}
$$

In particular, we will perform a complete bifurcation analysis with respect to the appeals $A_{1}$ and $A_{2}$.

\section{Analysis of the Model}

Let us first remark that if the individuals are completely indifferent one to each other when they first meet, i.e., if $x_{1}(0)=x_{2}(0)=0$, then the evolution of the feelings at the very beginning of the love story is fully determined by the appeals, because $\dot{x}_{i}(0)=A_{j}$. Thus, the love story has a positive start if and only if both individuals perceive the partner as appealing. As we will see later, this does not imply, however, that the love story cannot evolve positively in the long run even if it does not start in a promising way.

A second important remark is that limit cycles can not exist in model (1) because the diverge of the system (equal to $-\left(\alpha_{1}+\alpha_{2}\right)$ ) does not change sign. This excludes Hopf, tangent of limit cycles and homoclinic bifurcations [Kuznetsov, 2004]. In other words, the attractors of model (1) can only be equilibria.

\subsection{Robust vs. fragile couples}

The equilibria of model (1) are generically one or three. This can be easily understood from the shapes of the two isoclines $\dot{x}_{1}=0$ and $\dot{x}_{2}=0$ and from their dependence upon the appeals. Figure 2 shows the case where the two isoclines

$$
\begin{aligned}
& \dot{x}_{1}=0, \quad \text { i.e., } \quad x_{1}=\frac{1}{\alpha_{1}}\left(R_{1}\left(x_{2}\right)+A_{2}\right) \\
& \dot{x}_{2}=0, \quad \text { i.e., } \quad x_{2}=\frac{1}{\alpha_{2}}\left(R_{2}\left(x_{1}\right)+A_{1}\right)
\end{aligned}
$$


intersect at three different equilibria $x^{\prime}, x^{\prime \prime}$ and $x^{\prime \prime \prime}$ with $x^{\prime}<x^{\prime \prime}<x^{\prime \prime \prime}$. The analysis of the Jacobian reveals that $x^{\prime}$ and $x^{\prime \prime \prime}$ are stable nodes while $x^{\prime \prime}$ is a saddle. If the appeal $A_{1}$ is increased the (red) isocline $\dot{x}_{2}=0$ shifts upward until $x^{\prime}$ and $x^{\prime \prime}$ collide and disappear through a fold bifurcation. Similarly, if the appeal $A_{2}$ is increased the (blue) isocline $\dot{x}_{1}=0$ shifts to the right and $x^{\prime}$ and $x^{\prime \prime}$ collide and disappear through the same bifurcation. Conversely, if the appeals are reduced of a sufficient amount, $x^{\prime \prime}$ and $x^{\prime \prime \prime}$ collide and disappear through a second fold bifurcation. For particular parameter settings the two fold bifurcations can coincide at a codimension-2 cusp point which is identified by $x^{\prime}=x^{\prime \prime}=x^{\prime \prime \prime}$ as shown in Fig. 3. Notice from Fig. 3 that $A_{1}$ and $A_{2}$ must be of opposite sign at a cusp point.

The two fold bifurcation curves in the parameter space $\left(A_{1}, A_{2}\right)$ can be easily produced numerically through continuation and the typical result (obtained with the software package Auto [Doedel et al., 2007]) is shown in Fig. 4. Couples corresponding to points inside the region delimited by the fold bifurcation curves have two alternative stable states, namely $x^{\prime}$ and $x^{\prime \prime \prime}$, each one with its basin of attraction delimited by the stable manifold of the saddle $x^{\prime \prime}$. Couples of this kind are called fragile, because the quality of the romantic relationship can switch from high to low (i.e., from point $x^{\prime \prime \prime}$ to point $x^{\prime}$ ) or viceversa after a disturbance, e.g., after a temporary infatuation of one of the two individuals for another person. By contrast, couples corresponding to points outside the closed region in Fig. 4 are called robust, because they have a single globally stable equilibrium. In other words, romantic relationships in robust couples always return to the same quality after any disturbance or interaction with the rest of the world.

\subsection{High vs. low quality relationships}

Since positive values of $x_{i}$ represent positive feelings, ranging from sympathy to passion, while negative values are associated with antagonism and disdain, we can say that states with positive components represent high quality relationships, while states with negative components represent low quality relationships. In both cases these states will be called 
balanced, while states with components of opposite sign will be called unbalanced. It is therefore interesting to partition the space of the appeals into subregions giving rise to the same signs of the feelings at the stable equilibria $x^{\prime}$ and $x^{\prime \prime \prime}$. This can be done very easily by computing the curves in the space $\left(A_{1}, A_{2}\right)$ satisfying Eq. (1) with $\dot{x}_{1}=\dot{x}_{2}=0$ and $x_{1}=0$ or $x_{2}=0$, i.e.,

$$
R_{1}\left(A_{1} / \alpha_{2}\right)+A_{2}=0 \text { and } R_{2}\left(A_{2} / \alpha_{1}\right)+A_{1}=0
$$

and by eliminating those pieces of the curves on which the relationship $x_{1}=0$ or $x_{2}=0$ can be satisfied only at the saddle equilibrium $x^{\prime \prime}$. Thus, the pieces of the curves that must be eliminated are inside the bistability region of Fig. 4 where the saddle exists. The result is shown in Fig. 5, where the green and orange curves associated with a change of sign of one component of the stable equilibria $x^{\prime}$ or $x^{\prime \prime \prime}$ end at a point on the fold bifurcation at which the saddle $x^{\prime \prime}$ coincides with $x^{\prime}$ or $x^{\prime \prime \prime}$ and has a zero component.

Figure 5 shows that robust couples can experience high quality relationships in the long run even if one of the two individuals has a negative appeal. However, a low quality relationship is inevitable among robust couples if both individuals have negative appeals. Figure 5 also shows that fragile couples can be partitioned into seven classes. In the central region of Fig. 5 the two stable equilibria are balanced ( $x^{\prime}$ negatively and $x^{\prime \prime \prime}$ positively), in the two small regions touching the two cusp points both stable equilibria are unbalanced and in the remaining four regions one equilibrium is balanced and the other is unbalanced.

\subsection{Favorable vs. unfavorable evolution}

Robust couples have a globally stable equilibrium and therefore tend toward that equilibrium no matter what their initial state is. By contrast, fragile couples have two stable equilibria $x^{\prime}$ and $x^{\prime \prime \prime}$, with $x^{\prime \prime \prime}$ representing a more desirable relationship than $x^{\prime}$. It is therefore interesting to know if a fragile couple is inclined to converge to its most desirable attractor or not. This can be done if the attention is restricted to the most common situation, namely that 
involving individuals with unbiased feelings when they first meet, i.e., $x_{1}(0)=x_{2}(0)=0$. Thus, technically speaking, couples with favorable evolution are those for which the origin of the state space is in the basin of attraction of the equilibrium $x^{\prime \prime \prime}$. But in a fragile couple the boundary of the basin of attraction of $x^{\prime \prime \prime}$ is the stable manifold of the saddle, as shown in Fig. 6. Thus, the curve in the space of the appeals separating couples with favorable and unfavorable evolutions is the locus of the pairs $\left(A_{1}, A_{2}\right)$ for which the origin of the state space is on the stable manifold of the saddle. This allows one to produce this curve numerically through continuation as described in Appendix. The curve (obtained with the software package AUTO [Doedel et al., 2007]) is shown in pink in Fig.7: it lies entirely in the region of fragile couples (where the saddle $x^{\prime \prime}$ exists) and touch es generically the fold bifurcation curves at two points (notice that the case shown in Fig. 7 is non generic). As intuitively expected, high values of the appeals facilitate evolutions of the feelings toward the most desirable state $x^{\prime \prime \prime}$.

\subsection{Summary of the analysis}

Superimposing Figs. 4, 5, and 7, one obtains the complete catalog of the meaningful subclasses (i) $i=1, \ldots, 15$, of standard couples, shown in Fig. 8. The figure points out very clearly the role of the appeals. Indeed, increments of $A_{1}$ and/or $A_{2}$ are associated with transitions from poor to rich romantic relationships. For example, starting from a robust and low quality relationship (region (3)) and increasing $A_{1}$ and $A_{2}$, one can first transform the couple into a fragile couple with unfavorable evolution (region (8), then obtain a fragile couple with favorable evolution (region (13)) and finally end up in the class of robust and high quality relationships (region (1). This is in agreement with common wisdom and justifies, in a sense, the effort that men and women of all cultures generally make to become more attractive. 


\section{Conclusion and Call for Proposals}

We have extended in this paper previous studies performed on love dynamics [Rinaldi \& Gragnani, 1998] by considering also the case of non-appealing individuals. The analysis has been carried out by studying, through continuation, the bifurcations and other properties of a second-order non-linear system. The results are in agreement with common wisdom and show that improvements in the appeals give rise to improvements in the quality of the relationship. Moreover, it has been shown that individuals with negative appeal initially generate a negative reaction in the partner, but, despite this unfavorable start, the love story can evolve positively in the long run. The final result of the analysis is the complete catalog of possible behaviors of standard couples condensed in an easily interpretable diagram (Fig. $8)$.

The study would be more complete if we could present one convincing and well documented example for each possible behavior of our theoretically derived catalog. Examples could be taken from the technical literature, but in a sense they would be more appealing if they could be taken from the overwhelming number of popular novels, poems, films and songs dedicated to love stories. Since we are unable to do this at the moment, we take the liberty of asking the readers of this paper to help us by suggesting examples. Moreover, for creating a sort of homogeneous catalog of examples, we propose to limit the search to well known films.

Examples for the largest classes of couples, like those of robust couples, immediately come to mind. Titanic, a successful 1997 film starring Leonardo Di Caprio and Kate Winslet, is a good example of a robust and high quality couple (region (1) in Fig. 8). It describes the love story that develops on a ship between Jack and Rose while crossing the Atlantic Ocean. The story starts at the port of departure and ends only a few days later because Jack dies when the ship sinks after the collision with an iceberg. The two young lovers are so appealing and the level of intimacy they reach in a few days is so high that one can only imagine that this love story would end (if not dramatically interrupted) in a positive equilibrium. Actually, 
also the stability of this equilibrium is shown in the film, because Rose quickly returns to Jack after a short hesitation generated by the insinuations of a malicious pretender. Fatal attraction, a 1987 film starring Michael Douglas (Dan) and Gleen Close (Alex) is a good example of a robust couple with unbalanced feelings (region (4) in Fig. 8). Indeed, Dan and Alex are both physical very attractive but Alex has a very severe mental sickness. The result is that Dan has a positive appeal on her, while Alex has a negative appeal on him. In agreement with our theory, the relationship evolves toward an unbalanced state that ends, into tragedy.

Finding examples for the smallest regions of Fig. 8 is not as easy. In order to help the reader understanding the sense of this "call for proposals" we describe a possible example for one of the most narrow classes of our catalog, namely region (10) in Fig. 8. The story is described by E. Rostand in his masterpiece Cyrano de Bergerac, a "commèdie heroique" represented for the first time in Paris in 1897 and recently (1990) transformed by Jean-Paul Rappeneau into a film starring Gérard Depardieu (Cyrano de Bergerac), Anne Brochet (Roxane) and Vincent Perez (Christian de Neuvillette). Roxane, a beautiful and very romantic young lady, would not develop spontaneously positive feelings neither for Cyrano, a brave, sensitive, and well educated mousquetaire with a serious physical handicap (an abnormal nose), nor for Christian who is physically very attractive but crude and insensitive. This is why the two friends decide to "fuse" in a single person with the best characteristics of both: Cyrano suggests to his friend charming statements and romantic modes of behavior and writes to Roxane passionate letters that Christian signs. Roxane, obviously, falls in love with this very appealing though fictitious individual (the soul of Cyrano in the body of Christian) but, unfortunately she soon remains widow because Christian is accidentally killed. Since then, she melancholically refreshes her memory for her lover by reading every day the letters secretly written by Cyrano who is visiting her regularly and finally, understand that she is actually in love with him. All this is perfectly interpretable by imaging that Roxane and Cyrano are characterized by appeals $\left(A_{1}, A_{2}\right)$ of opposite signs falling in 
region (10). Indeed, this implies that Cyrano and Roxane can be in two alternative stable states, one positive $\left(x^{\prime \prime \prime}\right)$ reached at the end of their life and one unbalanced (whit $x_{1}^{\prime}<0$ and $x_{2}^{\prime}>0$ ). If Cyrano were to reveal his interest from the very beginning, i.e., when Roxane is not yet involved, then he would be refused because region (10) is in the region of fragile couples with unfavorable evolution. We can therefore conclude that he has been right in hiding his interest and in waiting for the most appropriate time to reveal himself. This is a very original and subtle way of saying that switching from an unfavorable state $\left(x^{\prime}\right)$ into the basin of attraction of a desirable state $\left(x^{\prime \prime \prime}\right)$ might be very difficult and long.

Thus, in conclusion, Titanic, Fatal attraction and Cyrano de Bergerac are films that could be associated, as shown in Fig. 9, to regions (1), (4) and (10) of our bifurcation diagram. Figure 9 is far from being complete and the reader is kindly invited to propose films that can ba associated to the still empty regions. In doing this, the reader should carefully exclude love stories of individuals with appeals that vary so much to involve sequentially two or more regions of Fig. 8. Indeed, in such cases the entire love story is more properly viewed as the concatenation of different love stories (corresponding to different appeals) separated by the events (a war, an accident, the discovery of a hidden aspect of the character of the partner, ...) that have caused the variation of the appeals. 


\section{References}

Allgower, E. L. \& Georg, K. [1990] Numerical Continuation Methods: An Introduction (Springer-Verlag, Berlin).

Ascher, U. M., Mattheij, R. M. M. \& Russell, R. D. [1995] Numerical Solution of Boundary Value Problems for Ordinary Differential Equations (SIAM, Philadelphia).

Bartholomew, K. \& Horowitz, L. M. [1991] "Attachment styles among young adults: a test of a four-category model," Journal of Personality and Social Psychology 61, 226-244.

Champneys, A. R., Kuznetsov, Yu. A. \& Sandstede, B. [1996] "A numerical toolbox for homoclinic bifurcation analysis," International Journal of Bifurcation and Chaos 6, 867887.

Doedel, E. J. [1981] "AUTO, a program for the automatic bifurcation analysis of autonomous systems," Congressus Numerantium 30, 265-384.

Doedel, E. J., Champneys, A. R., Dercole, F., Fairgrieve, T. F., Kuznetsov, Yu. A., Oldeman, B., Paffenroth, R. C., Sandstede, B., Wang, X. J. \& Zhang, C. H. [2007] AUTO-07p: Continuation and bifurcation software for ordinary differential equations, Department of Computer Science, Concordia University, Montreal, QC.

Doedel, E. J., Keller, H. B. \& Kernévez, J.-P. [1991] "Numerical analysis and control of bifurcation problems (II): Bifurcation in infinite dimensions," International Journal of Bifurcation and Chaos 1, 745-772.

Gragnani, A., Rinaldi, S. \& Feichtinger, G. [1997] "Cyclic dynamics in romantic relationships," International Journal of Bifurcation and Chaos 7, 2611-2619.

Griffin, D. W. \& Bartholomew, K. [1994] "Models of the self and other. Fundamental dimensions underlying measures of adult attachment," Journal of Personality and Social Psychology 67, 430-445. 
Iooss, G. \& Joseph, D. D. [1990] Elementary Stability and Bifurcation Theory (SpringerVerlag, New York), 2nd edn.

Kuznetsov, Yu. A. [2004] Elements of Applied Bifurcation Theory (Springer-Verlag, Berlin), 3rd edn.

Radzicki, M. J. [1993] "Dyadic processes, tempestuous relationships, and system dynamics," 9, 79-94.

Rinaldi, S. [1998a] "Laura and petrarch: An intriguing case of cyclical love dynamics," SIAM Journal on Applied Mathematics 58, 1205-1221.

Rinaldi, S. [1998b] "Love dynamics: The case of linear couples," Applied Mathematics and Computation 95, 181-192.

Rinaldi, S. \& Gragnani, A. [1998] "Love dynamics between secure individuals: A modeling approach," Nonlinear Dynamics, Psychology, and Life Sciences 2, 283-301.

Simpson, J. A., Gangestad, S. W. \& Lerma, M. [1990] "Perception of physical attractiveness: Mechanism involved in the maintenance of romantic relationships," Journal of Personality and Social Psychology 59, 1192-1201.

Sprott, J. C. [2004] "Dynamical models of love," Nonlinear Dynamics, Psychology, and Life Sciences 8, 303-314.

Strogatz, S. H. [1988] "Love affairs and differential equations," Mathematics Magazine 61, 35.

Strogatz, S. H. [1994] Nonlinear Dynamics and Chaos (Addison-Wesley, Reading, MA).

Wauer, J., Schwarzer, D., Cai, G. Q. \& Lin, Y. K. [2007] "Dynamical models of love with time-varying fluctuations," Applied Mathematics and Computation 188, 1535-1548. 


\section{Appendix}

In this Appendix we show that the locus of the pairs $\left(A_{1}, A_{2}\right)$ for which the origin of the state space is on the stable manifold of the saddle $x^{\prime \prime}$ (see Fig. 6) is a smooth curve that can be numerically obtained through standard continuation techniques [Allgower \& Georg, 1990].

The first step is to formulate the above geometric condition as a truncated two-boundaryvalue problem for model (1), i.e.,

$$
\begin{aligned}
\dot{x}_{1}+\alpha_{1} x_{1}-R_{1}\left(x_{2}\right)-A_{2} & =0 \\
\dot{x}_{2}+\alpha_{2} x_{2}-R_{2}\left(x_{1}\right)-A_{1} & =0 \\
x_{1}(0) & =0 \\
x_{2}(0) & =0 \\
-\alpha_{1} x_{1}^{\prime \prime}+R_{1}\left(x_{2}^{\prime \prime}\right)+A_{2} & =0 \\
-\alpha_{2} x_{2}^{\prime \prime}+R_{2}\left(x_{1}^{\prime \prime}\right)+A_{1} & =0 \\
\frac{d}{d x_{1}} R_{2}\left(x_{1}^{\prime \prime}\right) v_{2}-\lambda v_{1} & =0, \quad \lambda>0, \\
-\alpha_{1} v_{1}+\frac{d}{d x_{2}} R_{1}\left(x_{2}^{\prime \prime}\right) v_{1}-\alpha_{2} v_{2}-\lambda v_{2} & =0 \\
v_{1}^{2}+v_{2}^{2}-1 & =0 \\
\left(x_{1}(T)-x_{1}^{\prime \prime}\right) v_{1}+\left(x_{2}(T)-x_{2}^{\prime \prime}\right) v_{2} & =0
\end{aligned}
$$

where Eqs. (Aa, b) define the trajectory in the finite time interval $[0, T]$, Eqs. (Ac, d) fix the initial condition, Eqs. (Ae, f) identify the saddle $x^{\prime \prime}$, Eqs. (Ag-i) define the unstable left eigenvector $v$ of the Jacobian of model (1) at $x^{\prime \prime}$, and Eq. (Aj) imposes that the distance between the terminal point at time $T$ and the saddle $x^{\prime \prime}$ is orthogonal to $v$. Since $v$ is orthogonal to the stable right eigenvector of the Jacobian (see, e.g., [Iooss \& Joseph, 1990]), Eq. (Aj) imposes that $x(T)$ lies along the linearized stable manifold of the saddle $x^{\prime \prime}$. Overall, we have 10 smooth conditions involving 11 variables, $x_{1}(0), x_{2}(0), x_{1}(T), x_{2}(T), x_{1}^{\prime \prime}, x_{2}^{\prime \prime}, v_{1}, v_{2}$, 
$\lambda, A_{1}, A_{2}$, while $T$ must be considered as a constant parameter. Thus, problem (A) has one degree of freedom, namely it defines a one-dimensional smooth manifold of solutions, that projected onto the space $\left(A_{1}, A_{2}\right)$ gives a smooth curve for each value of $T$. For $T$ sufficiently large, such a curve converges (uniformly) to our desired curve. This is the standard technique used to approximate homoclinic and heteroclinic bifurcation curves [Champneys et al., 1996].

The second step is to find a pair $\left(A_{1}, A_{2}\right)$ for which the solution of problem $(\mathrm{A})$ is known. This is easy, since for $A_{1}=A_{2}=0$ the saddle $x^{\prime \prime}$ is at the origin, so that $x(t)=0$ for all $t \in[0, T]$ is the solution.

The third step is to discretize the boundary-value problem (A) into a purely algebraic continuation problem. This is done automatically by most of the advanced software packages for continuation, by means of the so-called orthogonal collocation method (see, e.g., [Ascher et al., 1995; Doedel et al., 1991]). Among these packages, we used Auto [Doedel, 1981] (see [Doedel et al., 2007] for the currently updated version), since it is the only package allowing user-defined boundary-value problems.

The last step is the interpretation of the numerical results. As shown in Fig. 7, our curve (pink in the figure) is delimited by two points of tangent contact with the fold bifurcation curves. Such points depend smoothly upon the other model parameters and can be on the same fold bifurcation or on different ones. For critical parameter combinations, the contact points can coincide with one or both the cusp points (the case in Fig. 7 is intentionally nongeneric, since one of the contact points coincides with the right cusp point).

Note that the contact points generically divide the fold bifurcation curves into two or three pieces (three in the case of two contact points on the same bifurcation). Along the pieces (if any) of the lower fold bifurcation delimiting the region of favorable evolution, and along those pieces (if any) of the upper bifurcation delimiting the region of unfavorable evolution (see, e.g., the piece next to the left cusp point in Fig. 7), the trajectory starting from the origin reaches the saddle-node equilibrium, which is however an unstable equilibrium point. 


\section{Figure captions}

\section{Figure 1}

Typical reaction functions $R_{1}\left(x_{2}\right)$ (blue) and $R_{2}\left(x_{1}\right)$ (red) of two secure and non-synergic individuals. The two graphs correspond to

$$
R_{1}\left(x_{2}\right)=\frac{e^{x_{2}}-e^{-x_{2}}}{e^{x_{2}} / R_{1}^{+}-e^{-x_{2}} / R_{1}^{-}} \quad \text { and } \quad R_{2}\left(x_{1}\right)=\frac{e^{x_{1}}-e^{-x_{1}}}{e^{x_{1}} / R_{2}^{+}-e^{-x_{1}} / R_{2}^{-}}
$$

with $R_{1}^{+}=1, R_{2}^{+}=2$, and $R_{1}^{-}=R_{2}^{-}=-1$.

\section{Figure 2}

The isoclines $\dot{x}_{1}=0$ (blue) and $\dot{x}_{2}=0$ (red) in the case of three equilibria $x^{\prime}, x^{\prime \prime}$ and $x^{\prime \prime \prime}$. The figure is drawn for the case with $R_{1}$ and $R_{2}$ as in Fig. 1 and $\alpha_{1}=0.1, \alpha_{2}=0.3$, and $A_{1}=A_{2}=0.3$.

\section{Figure 3}

Codimension-2 bifurcation (cusp) characterized by $x^{\prime}=x^{\prime \prime}=x^{\prime \prime \prime}$. Parameter values as in Fig. 2, except $A_{1}=-2.38$ and $A_{2}=1.128$.

\section{Figure 4}

Fold bifurcation curves of model (1) for $R_{1}$ and $R_{2}$ as in Fig. 1 and $\alpha_{1}=0.1, \alpha_{2}=0.3$. $C_{1}$ and $C_{2}$ are cusp points. Inside the closed region (fragile couples) the model has two stable equilibria and one saddle, while outside (robust couples) it has only one stable equilibrium.

\section{Figure 5}

The signs of the feelings at the stable equilibria $x^{\prime}$ and $x^{\prime \prime \prime}$ change from negative to positive crossing the green and orange curves (corresponding to Eq. 2) in the direction of the arrows. 
Parameter values as in Fig. 4.

\section{Figure 6}

State portrait of a fragile couple with two stables states ( $x^{\prime}$ and $x^{\prime \prime \prime}$ in green) and a saddle ( $x^{\prime \prime}$ in blue). The stable manifold of the saddle (thick trajectory) is the boundary of the basins of attraction of the two stable states (white and gray regions). Since the origin (state of indifference) is in the basin of attraction of $x^{\prime \prime \prime}$ the love story evolves toward the positive state. Reaction functions are as in Fig. 1 with $\alpha_{1}=0.1, \alpha_{2}=0.3, A_{1}=0.3$, and $A_{2}=0.2$.

\section{Figure 7}

Fragile couples partitioned into couples with favorable and unfavorable evolution. Parameters and functions as in Fig. 4.

\section{Figure 8}

The complete catalog of meaningful subclasses of standard couples. Robust couples are positively balanced in region (1), negatively balanced in region (3), and unbalanced in regions (2) and (4). Fragile couples have favorable evolutions in regions (12)-(15) and unfavorable in regions (5)-(11) (see the side panel where the dashed rectangle is stretched and magnified). The two stable equilibria $x^{\prime}$ and $x^{\prime \prime \prime}$ of fragile couples lie in the quadrants (I-IV) reported in the legend where the equilibrium reached from the state of indifference is indicated in green. Parameters and functions as in Fig. 4.

\section{Figure 9}

A love story described in a film can be associated to every region of the bifurcation diagram in Fig. 8. For example, Titanic, Fatal attraction, Cyrano de Bergerac are here associated to regions (1), (4) and (10) of the bifurcation diagram. The reader is invited to propose films for the still empty regions. 




love of the partner

Figure 1 


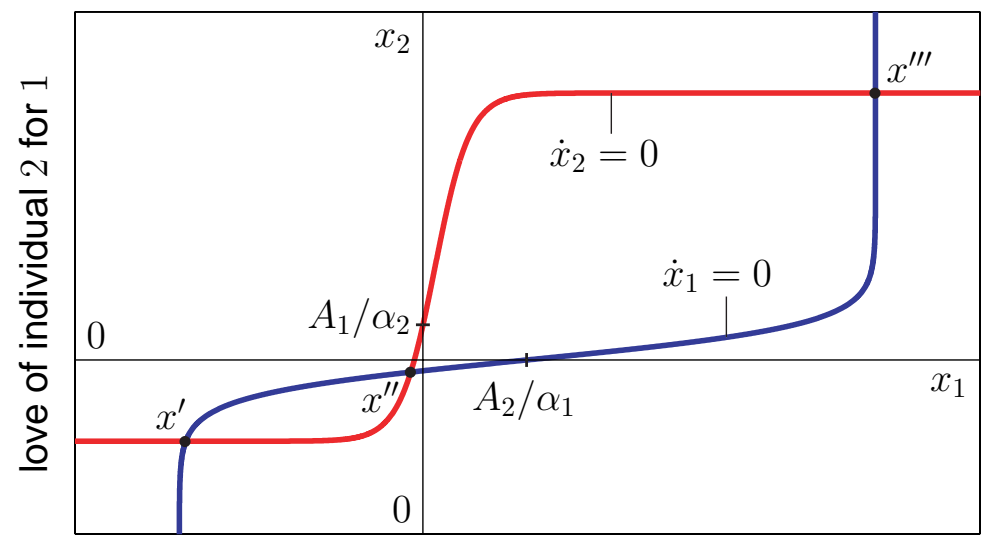

love of individual 1 for 2

Figure 2 


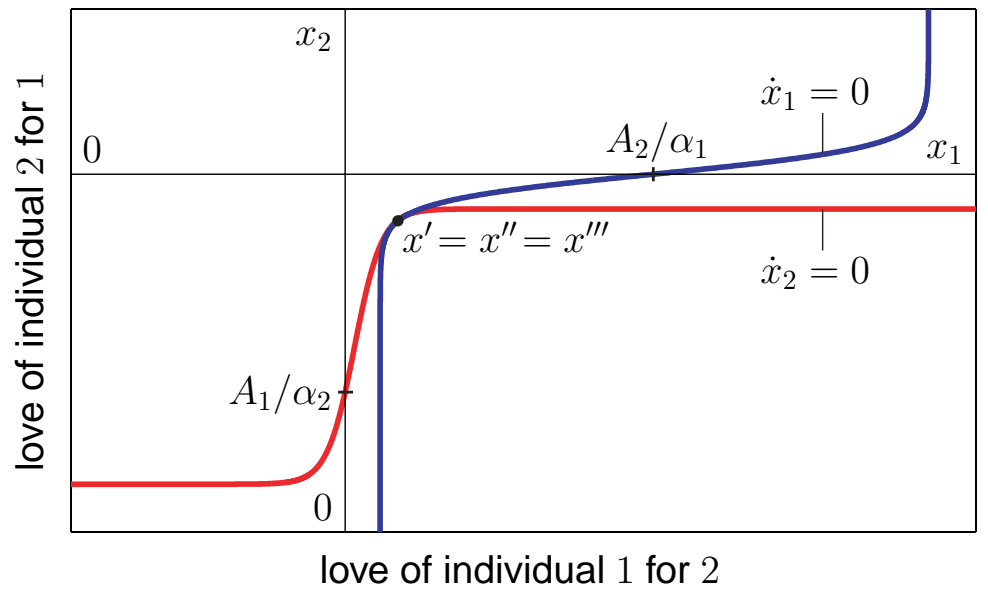

Figure 3 


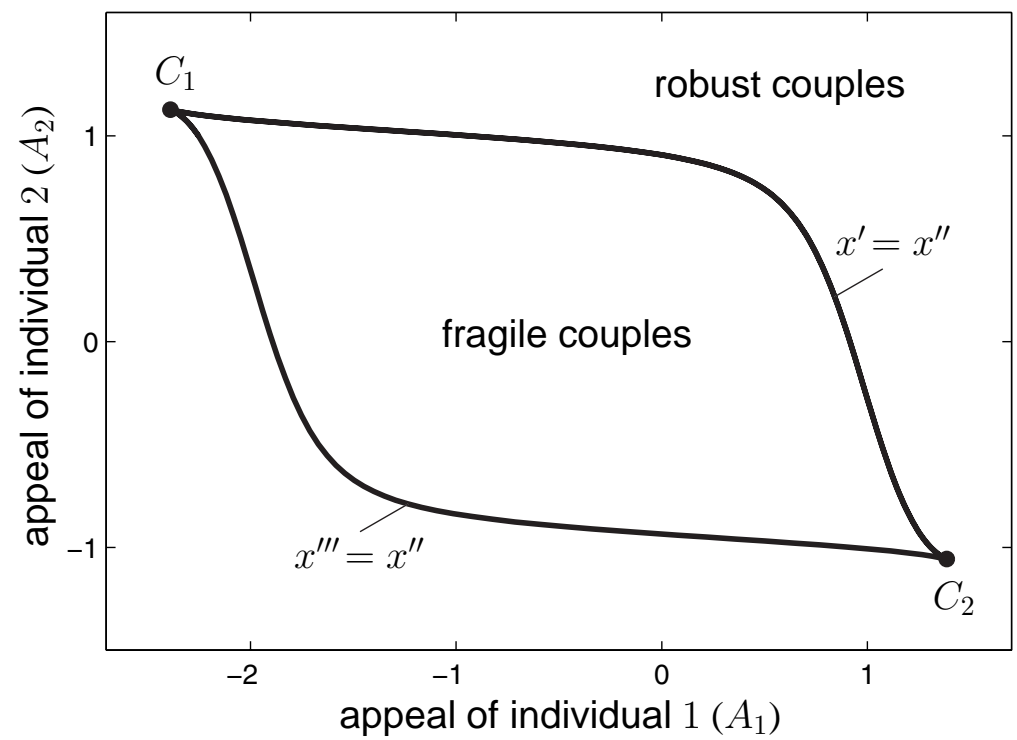

Figure 4 




Figure 5 




Figure 6 




Figure 7 


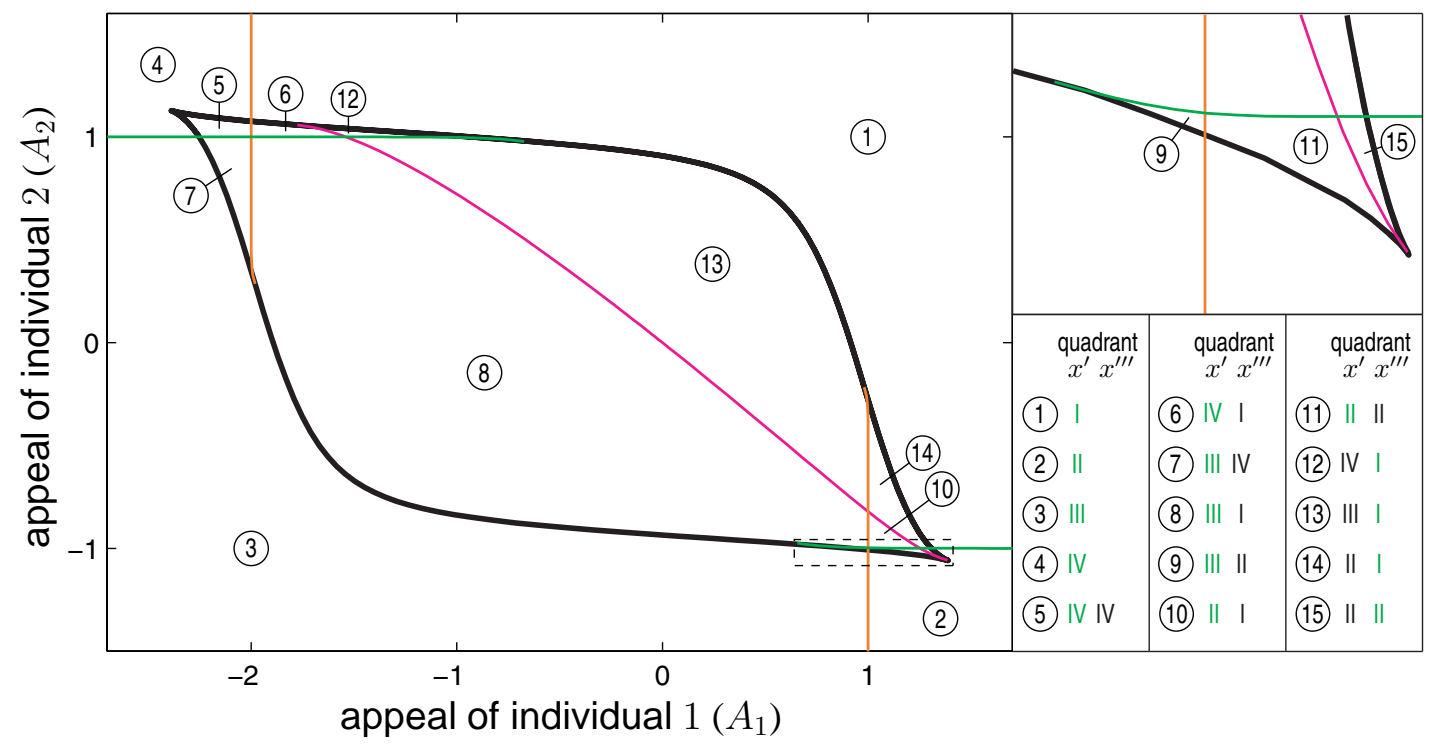

Figure 8 


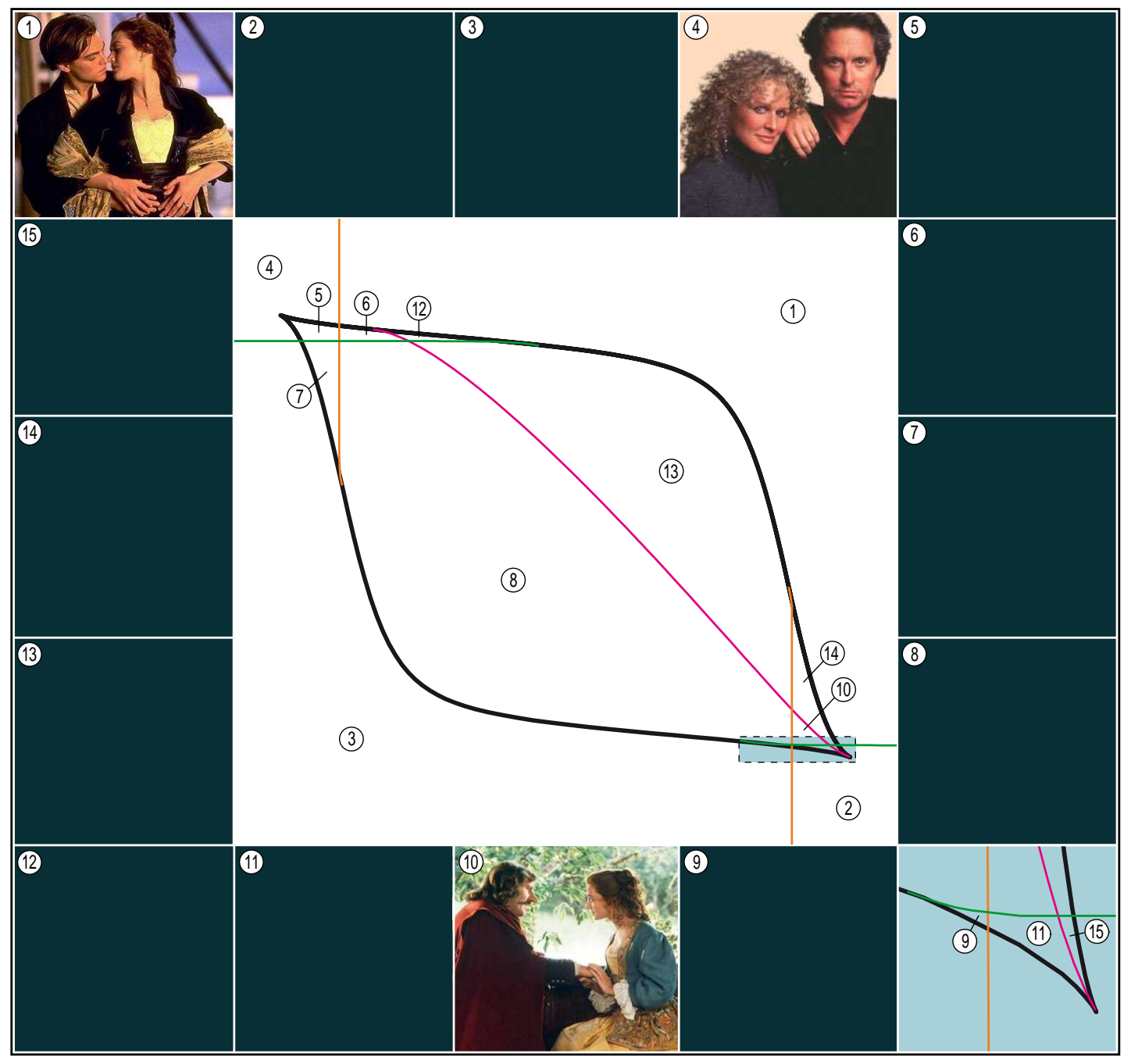

Figure 9 